LOS SENTIDOS DEL COMPROMISO SOCIAL UNIVERSITARIO. UNA APROXIMACIÓN A LA CONSTRUCCIÓN DEL ESTADO DEL ARTE. Páginas 129-146 en Revista de la Escuela de Ciencias de la Educación, año 10, número 9, enero a diciembre de 2014. ISSN 1851-6297. ISSN EN LINEA 2362-3349.

\title{
LOS SENTIDOS DEL COMPROMISO SOCIAL UNIVERSITARIO. UNA APROXIMACIÓN A LA CONSTRUCCIÓN DEL ESTADO DEL ARTE
}

\author{
Por Carina Andrea Gerlero \\ (Universidad Nacional de Rosario-Ministerio de Educación de Santa Fe)*, \\ Argentina. \\ cgerlero@hotmail.com
}

Recibido: 04/07/2014 Aceptado: 04/11/2014

\section{Resumen}

El presente artículo (1) tiene como objetivo identificar y analizar las perspectivas teóricas sobre el Compromiso Social Universitario (CSU) a partir del relevamiento del conocimiento producido en el período 2007-2013, con el propósito de precisar qué se entiende por CSU y a qué modelo de Universidad suscribe. Se indagan relaciones entre éste y la tradición reformista de las universidades públicas argentinas, principalmente en lo referente a su función social. Se analizan las similitudes y diferencias entre el CSU y la Responsabilidad Social Universitaria (RSU), tratando de discernir si se trata de una cuestión semántica o si se sostienen en diferentes valores y conceptos.

Se identifican algunos principios obligados al momento de referirse al CSU, tales como: la definición de proporcionar educación universitaria de calidad igual para todos; la democratización y la orientación solidaria de las acciones universitarias, a través de las funciones de docencia, extensión, investigación y gestión; la necesidad de establecer un diálogo con la sociedad, para contribuir, conjuntamente, a desarrollar la cultura y los procesos de integración regional, disminuir la exclusión y brechas de desigualdad, profundizar la democracia y el bien común, asumir un compromiso ético con la transformación y construcción social.

\section{Palabras Clave}

Universidad - Función social - Democratización - Diálogo - Compromiso ético.

* Subsecretaria de Planificación y Articulación Educativa. Ministerio de Educación de la Provincia de Santa Fe. Especialista en Política y Gestión de la Educación Superior (CEIUNR). Doctoranda en Educación en Ciencias Experimentales (FBCB - UNL). 
Revista de la Escuela de Ciencias de la Edducación, año 10, número 9, enero a diciembre de 2014. Páginas 129-146. ISSN 1851-6297. ISSN EN LINEA 2362-3349. LOS SENTIDOS DEL COMPROMISO SOCIAL UNIVERSITARIO. UNA APROXIMACIÓN A la construcción del estado del arte. Carina Andrea Gerlero.

\begin{abstract}
The object of this article is to identify and analyze the theoretical perspectives about the University Social Commitment (USC) based on the knowledge gathered during the period 2007-2013, to precise the meaning of UCS and to which model of University it subscribes. The relationship between USC and the tradition of reforms of the Argentine public Universities is assessed; mainly with regard to its social role. The similarities and differences between the USC and the University Social Responsibility (USR) are analyzed in order to decide if there is a semantic matter involved or if there is a difference in values and concepts.

Some significant principles are observed when dealing with USC, such as: the definition of university education of equal quality for everyone; the democratization and the charitable aspect of the university through the roles of teaching, extension, research and management and the need to establish a dialogue with society to contribute, together, to develop culture and processes of regional integration, to reduce exclusion and the gap between social groups, to deepen democracy and common welfare and to make an ethic commitment to social transformation and construction.
\end{abstract}

\title{
Key Words
}

University - Social role - Democratization - Dialogue - Ethic commitment.

\section{Acerca de la constitución del campo del Compromiso Social Universitario}

En América Latina las universidades públicas se sustentan y organizan, simultáneamente, en base a dos grandes modelos: el de la Universidad profesionalizante y el de la Universidad científica, que asumen una impronta propia producto de las ideas provenientes de la Reforma del '18. Desde sus postulados, se inician procesos de democratización de las IES, involucramiento de los universitarios con las problemáticas políticas y sociales del país, relación entre las universidades de Latinoamérica e innovación de los programas académicos. Progresivamente, las Universidades Reformistas adhieren al libre acceso y la gratuidad, a la libertad de cátedra, a los concursos para el acceso a los cargos docentes, a la autonomía y autarquía universitaria, a la participación de los claustros -docente, estudiantil y de graduados- en el gobierno de la universidad y de las facultades, a la extensión universitaria; a la modernización de las estrategias pedagógicas de los docentes y planes de estudios, entre otros.

Este modelo de Universidad Reformista se instala en los actores universitarios, pero no se desarrolla de manera continuada a lo largo de la historia, en términos generales se ve potenciado en los períodos democráticos y recortado en los períodos donde el gobierno se instala a través de dictaduras. Desde ese momento hasta el presente, en los períodos democráticos las universidades han profundizado su vínculo con la sociedad, desde distintos formatos, modelos o 
Revista de la Escuela de Ciencias de la Educación, año 10, número 9, enero a diciembre de 2014. Páginas 129-146. ISSN 1851-6297. ISSN EN LÍNEA 2362-3349. LOS SENTIDOS DEL COMPROMISO SOCIAL UNIVERSITARIO. UNA APROXIMACIÓN A la construcción del estado del arte. Carina Andrea Gerlero.

estrategias, que no se abordarán sino tangencialmente en el presente trabajo, ya que el recorte temporal excede los límites del mismo.

A partir del análisis de los documentos y bibliografía disponible entre 2007 y 2013, es posible observar que en los últimos años asistimos a la configuración de un campo de producción especializada de conocimiento en la Educación Superior (ES), referido al CSU. Se identifican ciertos acontecimientos, sucedidos en los años 2007/2008 que parecieran determinar la emergencia de este campo. A nivel de América Latina y el Caribe, evidentemente ocupa un lugar central la Conferencia Regional de Educación Superior (CRES), realizada en 2008, dado que delimita las perspectivas para la ES en la región. Los posicionamientos de la CRES, junto con los surgidos de las conferencias de otras regiones, son parte de los insumos que se ponen en juego en la Conferencia Mundial de Educación Superior (CMES) desarrollada en París, en el año 2009. Estas perspectivas posibilitan la comprensión de los sentidos del CSU y, por otra parte, enriquecen las producciones teóricas, debates, congresos e investigaciones al tiempo que facilitan la configuración del CS, con entidad propia, en el campo de la ES universitaria.

Es de interés repasar los planteamientos surgidos en distintas instancias de diálogo en torno a los temas universitarios que configuran los antecedentes de las Conferencias arriba mencionadas. En este sentido, se sitúa la CRES del año 1996 realizada en La Habana y la CMES del año 1998, en éstas se toma en cuenta entre otros aspectos, el documento elaborado por la UNESCO en 1995, en relación a "Orientación sobre cambio y desarrollo en la educación superior". A partir de este documento, surge la necesidad de fortalecer la equidad, la calidad, la pertinencia y la internacionalización en el campo de la ES. En estas instancias se observa la influencia de las políticas propias de los '90 y, si bien se visualizan posicionamientos que atañen a la relación de la Universidad con la sociedad, no surge aún la estructuración de éstos en términos de CSU.

Más recientemente, se puede mencionar el Congreso Internacional de Rectores de América Latina y el Caribe: "EL COMPROMISO SOCIAL DE LAS UNIVERSIDADES DE AMÉRICA LATINAY EL CARIBE”, realizado en la Universidad Federal de Mina Gerais, en septiembre de 2007. En la declaración emitida por los Rectores participantes (2007:2), se plantea "Concebir por compromiso social de las universidades (...) la implementación de políticas institucionales que tengan por principio a la educación como derecho humano, bien público, socialmente referenciada, en consonancia con los valores de calidad, pertinencia, relevancia, inclusión y equidad". Por otra parte, reconocen que el abordaje realizado en la CMES de 1998 en torno a la pertinencia de la ES les permite pensar al CSU en relación a los alcances de una educación socialmente referenciada, entendiendo que para tal propósito se requiere la construcción de saberes que puedan contribuir a disminuir las desigualdades potenciadas a partir de los procesos de globalización y garantizar los derechos que contribuyen a 
Revista de la Escuela de Ciencias de la Educación, año 10, número 9, enero a diciembre de 2014. Páginas 129-146. ISSN 1851-6297. ISSN EN LINEA 2362-3349. LOS SENTIDOS DEL COMPROMISO SOCIAL UNIVERSITARIO. UNA APROXIMACIÓN A la construcción del estado del arte. Carina Andrea Gerlero.

una mejor calidad de vida. También proponen diferentes temáticas para abordar en la agenda universitaria, tales como el compromiso con la expansión de la cobertura atendiendo a las necesidades de inclusión social con calidad, no incluir a la ES en los acuerdos del GATS (Acuerdo General sobre el Comercio de Servicios) y la promoción de una internacionalización solidaria de la ES en América Latina y el Caribe, entre otros. Pero la perspectiva central de la agenda reside en el planteo de "promover un salto cualitativo en el compromiso social de las Universidades, estimulando la producción de cambios estratégicos", de manera tal que se pase desde acciones aisladas, de bajo perfil y escasa sistematización a líneas programáticas que sean parte de las misiones de las Instituciones de Educación Superior (IES).

En el marco de este Congreso de Rectores, se destaca la conferencia de Miguel Rojas Mix sobre "El Compromiso Social de las Universidades de América Latina y el Caribe". En la misma, plantea que la base del CSU es proporcionar educación de calidad, en condiciones de igualdad, para todos los ciudadanos. Específicamente, refiriere al CSU como:

(...) un compromiso amplio que se extiende sobre diversos campos vinculados a la reproducción y perfeccionamiento del modelo social: la equidad, la ciencia, la eficiencia profesional, la cultura y la identidad, el pluralismo ideológico, la ética social, la conservación de la memoria histórica y de la universalidad del saber, y la creación de masa crítica (Rojas Mix, 2007:179).

Asimismo, señala los siguientes aspectos como roles de la Universidad para lograr este compromiso: desarrollar la cultura y la integración cultural, entendiendo que la memoria histórica forja el imaginario en la construcción de la identidad y posibilita el pensamiento crítico en un mundo globalizado; orientar su acción al bien común, al servicio de una nación, lo que otorga pertinencia a la $\mathrm{ES}$, en tanto permite contextualizar y discernir que conviene a su cultura y que fortalece su desarrollo; formar en valores, desarrollar y defender la democracia, de manera tal que se permita rescatar la relación que tienen la democracia -en el campo político- y la filosofía -en el campo del conocimiento-, con el saber vivir y pensar racionalmente en sociedad; disminuir las brechas y las desigualdades que atentan contra la justicia social, dejando de lado la tendencia liberal que propone sustituir la democracia por el mercado y lo político por lo económico.

Rojas Mix (2007) sostiene que el desarrollo del CSU requiere de la construcción de diálogos permanentes de la Universidad con distintos actores sociales de orden local, nacional y regional. En este último plano, también cobra valor el rol de la Universidad en garantizar la igualdad de oportunidades para el acceso a los estudios superiores y una educación en valores, que favorezca el pensamiento crítico y, a su vez, defienda y construya derechos individuales y colectivos. 
Revista de la Escuela de Ciencias de la Educación, año 10, número 9, enero a diciembre de 2014. Páginas 129-146. ISSN 1851-6297. ISSN EN LÍNEA 2362-3349. LOS SENTIDOS DEL COMPROMISO SOCIAL UNIVERSITARIO. UNA APROXIMACIÓN A la construcción del estado del arte. Carina Andrea Gerlero.

En el mencionado Congreso Internacional de Rectores de América Latina y el Caribe, también se desarrolló la conferencia del Rector de la Universidad Autónoma de Santo Domingo, Roberto Tejada Reyna. En la exposición, titulada "Propuestas para una agenda relativa al Compromiso Social de la Universidad", Tejada Reyna (2007) formula que la propia misión y filosofía de la Universidad, la sitúan como una institución con CS. En relación a la agenda, se destacan los siguientes planteos: la necesidad de construirla en consonancia con la agenda social, dado que esto le otorgará sentido y pertinencia a las acciones universitarias; asumir el compromiso con la formación de profesionales con sentido humano y con valores; desarrollar un currículum universitario que contribuya a mejorar la calidad de vida de los ciudadanos; ser parte activa en la efectivización de las metas de desarrollo del milenio planteadas por la UNESCO, principalmente en lo referente a propiciar una redistribución equitativa de la riqueza y a la erradicación de la pobreza, el hambre, el analfabetismo, la mortalidad infantil, entre otros.

En noviembre del 2007 en Bogotá, Colombia, se lleva a cabo el IX Congreso Iberoamericano de Extensión Universitaria, cuya conferencia central "EI Compromiso Social de las Universidades", estuvo a cargo de Carmen García Guadilla. En la misma refiere al CSU como inherente a los fines de la Universidad $y$ analiza las distintas formas de entenderlo y denominarlo, fundamentalmente a partir de mediados del siglo XX hasta la actualidad.

Durante su exposición plantea que en las décadas del ‘50 y ’60 el CSU representaba la creencia de la ideología desarrollista, acerca de que la educación permitiría superar el subdesarrollo de los países en esta situación, la premisa era que con mayor educación se lograría mayor productividad y mayor movilidad social. Expresa que por tal motivo algunos autores denominaron a este período la "edad de la inocencia", dado que solamente la educación no puede cubrir ese rol. Rescata el posicionamiento de Paulo Freire cuando defiende la idea de extensión universitaria como proceso de comunicación dialógica y horizontal entre la Universidad y la sociedad, en contraposición al formato unidireccional, donde las universidades "dan" y la sociedad "recibe".

En los '70, sitúa la "edad del escepticismo", destaca el surgimiento de las teorías críticas socioeducativas, donde influye el pensamiento reproductivista de Bordieu y Passeron y la idea de Darcy Ribeiro, que propone suplantar la "universidad tradicional" por la "universidad necesaria", que propicie el compromiso de la Universidad con la sociedad. La década del '80 se caracteriza como "década perdida" debido a las crisis económicas acaecidas en los comienzos del proceso de globalización, entiende que disminuyeron las influencias de las teorías críticas y surgen algunos enfoques de acción participativa, que en la ES se relacionan con la producción y distribución de conocimiento en relación al entorno social. En los '90, destaca que se reivindican los enfoques de Freire, Echavarría, Ribeiro y otros referentes latinoamericanos ante los nuevos para- 
Revista de la Escuela de Ciencias de la Educación, año 10, número 9, enero a diciembre de 2014. Páginas 129-146. ISSN 1851-6297. ISSN EN LINEA 2362-3349. LOS SENTIDOS DEL COMPROMISO SOCIAL UNIVERSITARIO. UNA APROXIMACIÓN A la construcción del estado del arte. Carina Andrea Gerlero.

digmas de la ciencia. En este período se cuestiona la certeza y el determinismo y se valoriza la subjetividad social, los actores sociales, la democracia como "expansión de la conciencia reflexiva en todos los espacios de interacción social". Esto posibilita por una parte una nueva comprensión del mundo, basada en la diversidad, el pluralismo, los valores de la solidaridad, responsabilidad, respeto mutuo y, por otra parte, le otorga valor a la producción de conocimientos contextualizados, integrados e interdisciplinarios. No obstante, en paralelo, en esta década también se instalan las políticas neoliberales de ES.

Finalmente, caracteriza al siglo XXI como "la edad del compromiso social", entendiéndolo como un compromiso con el desarrollo humano y sustentable para hacer frente a la globalización y a las desigualdades que ésta genera. Expresa que:

El compromiso social como exigencia es uno de los elementos cruciales de este final de la primer década de este siglo. Este compromiso social debe superar las limitaciones que tiene el concepto de extensión, reivindicando la necesaria contextualización de los conocimientos, la interacción con la sociedad, los procesos de aprendizaje compartidos y orientados hacia la transdisciplinariedad, procesos interactivos de la educación con la sociedad cada vez más viables, reivindicando las redes como organizaciones de aprendizaje colectivo, a la educación sin fronteras de ningún tipo (García Guadilla, 2008:134).

Es de destacar que estas instancias de encuentro que tuvieron lugar a nivel regional, confluyen en dos grandes Conferencias. La Conferencia Regional de ES de América Latina y el Caribe, llevada a cabo en junio el año 2008, en Cartagena de Indias y la Conferencia Mundial de ES: "La nueva dinámica de la educación superior y la investigación para el cambio social y el desarrollo", realizada en París, en julio el 2009.

En la Declaración Final de la CRES (2008:1) se expresa que el objetivo central reside en "configurar un escenario que permita articular, de forma creativa y sustentable, políticas que refuercen el compromiso social de la Educación Superior, su calidad y pertinencia, y la autonomía de las instituciones". En relación al CSU, se destacan los siguientes ejes:

- Su posicionamiento en relación al carácter humanista de la Educación Superior y la consecuente orientación a la formación integral de personas, ciudadanos y profesionales, capaces de abordar con responsabilidad ética, social y ambiental las necesidades del contexto y participar activa, crítica y constructivamente en la sociedad.

- La promoción del respeto y la defensa de los derechos humanos, haciéndolos presentes en programas de formación, así como en las prioridades de investigación, extensión y cooperación interinstitucional. 
Revista de la Escuela de Ciencias de la Educación, año 10, número 9, enero a diciembre de 2014. Páginas 129-146. ISSN 1851-6297. ISSN EN LÍNEA 2362-3349. LOS SENTIDOS DEL COMPROMISO SOCIAL UNIVERSITARIO. UNA APROXIMACIÓN A la construcción del estado del arte. Carina Andrea Gerlero.

- La definición de avanzar en una relación más activa de las Universidades con sus contextos.

- La vinculación de la calidad a la pertinencia y la responsabilidad con el desarrollo sostenible de la sociedad.

- La necesidad de un modelo académico que se caracterice por la indagación de los problemas en sus contextos; la producción y transferencia del valor social de los conocimientos; el trabajo conjunto con las comunidades; una investigación científica, tecnológica, humanística y artística fundada en la definición explícita de problemas a atender, de solución fundamental para el desarrollo del país o la región, y el bienestar de la población; una activa labor de divulgación, vinculada a la creación de conciencia ciudadana sustentada en el respeto a los derechos humanos y la diversidad cultural; un trabajo de extensión que enriquezca la formación, colabore en detectar problemas para la agenda de investigación y cree espacios de acción conjunta con distintos actores sociales, especialmente los más postergados.

Por su parte en el comunicado de la CMES del año 2009 también se visualizan posturas que tienen que ver con el CSU o la RSU, al referir a:

- La responsabilidad social de la educación superior en hacer avanzar nuestra comprensión de problemas polifacéticos con dimensiones sociales, económicas, científicas y culturales, así como nuestra capacidad de hacerles frente.

- La educación superior debe no sólo proporcionar competencias sólidas para el mundo de hoy y de mañana, sino contribuir además a la formación de ciudadanos dotados de principios éticos, comprometidos con la construcción de la paz, la defensa de los derechos humanos y los valores de la democracia.

- La convocatoria a los Estados Miembros, en colaboración con todas las partes interesadas, a elaborar políticas y estrategias en el plano de los sistemas $y$ de los establecimientos con el fin de garantizar inversiones adecuadas en educación superior e investigación, en sintonía con las necesidades y expectativas crecientes de la sociedad.

Los aportes realizados por los asistentes a estas Conferencias, Encuentros, Congresos, ya sea en calidad de expositores o de representantes de las Universidades, coadyuvó en el inicio de la configuración del campo del CSU. Además, al ser asumido el tema por los máximos responsables de las IES, facilita su presencia en la agenda universitaria y sienta una postura clara en el plano declarativo, por tratarse de instancias de diálogo. Asimismo, es de sumo valor tanto el llamado a la acción que éstos realizan, así como el compromiso institucional que asumen desde las distintas funciones universitarias, para el desarrollo de políticas concretas que se piensen desde el compromiso social, en cada uno de los países y de los territorios donde se insertan sus acciones. La perspectiva del CSU se instaló en la agenda universitaria a partir de estos encuentros y sigue presente en la actualidad a través de distintas instancias 
Revista de la Escuela de Ciencias de la Educación, año 10, número 9, enero a diciembre de 2014. Páginas 129-146. ISSN 1851-6297. ISSN EN LINEA 2362-3349. LOS SENTIDOS DEL COMPROMISO SOCIAL UNIVERSITARIO. UNA APROXIMACIÓN A la construcción del estado del arte. Carina Andrea Gerlero.

y acciones que desarrollan las IES en las amplias y complejas temáticas que abarca el CSU, reforzando la idea acerca de que es un compromiso inherente a la Universidad y que constituye un principio político e ideológico que atraviesa a todas las funciones universitarias.

Se observa que la relación que se establece entre el CSU y los valores sociales y culturales de la educación, se centra en el propósito de disminuir los impactos negativos de la globalización y otras políticas neoliberales. Por otra parte, se pretende contribuir a la disminución de las desigualdades que atraviesan a nuestras sociedades, a partir de la posibilidad de ampliar la participación de los ciudadanos en la toma de decisiones sobre las problemáticas y situaciones de las que son parte. El acceso de más jóvenes a una formación universitaria integral, un modelo educativo humanístico, el desarrollo de investigaciones pertinentes a las necesidades sociales, prácticas de extensión que propicien el diálogo entre la Universidad y la sociedad, constituyen la base para lograr estos propósitos.

\section{Los enfoques teóricos sobre el Compromiso Social Universitario y la Responsabilidad Social Universitaria}

EI CSU es concebido de manera polisémica, con múltiples y diferentes significados. En el presente apartado, se pretende analizar los enfoques y las concepciones que se ponen en juego con el propósito de comprender el sentido que se le otorga, es decir comprender de qué hablamos cuando hablamos de CSU. Podemos pensar que estas distintas formas de concebir al CSU se asocian a diferentes formas de entender a la Universidad, así como a sus misiones y las acciones que definen las funciones esenciales que deben desarrollarse en las instituciones universitarias. Precisar el sentido del CS interpela a señalar el posicionamiento que subyace en relación a la "Idea de Universidad" y los sentidos que se otorga a la ES universitaria.

En un intento de aportar a este análisis, es interesante agrupar los conceptos que emergen en función de las perspectivas teóricas que subyacen y observar cómo juegan en ellas los demás aspectos del CSU. En este sentido, se abordarán por un lado las concepciones que refieren al CSU o a la RSU como una estrategia que se sustenta en la función social de la Universidad y a la misión indelegable de las Universidades en la construcción de las sociedades en las que se insertan. Por otro lado, se analizarán las concepciones constituidas desde una lógica más cercana a la responsabilidad social empresaria (RSE) y que, consecuentemente, relacionan a la RSU con el rol de la Universidad en la gestión de impactos, evaluación y rendición de cuentas. 
Revista de la Escuela de Ciencias de la Educación, año 10, número 9, enero a diciembre de 2014. Páginas 129-146. ISSN 1851-6297. ISSN EN LÍNEA 2362-3349. LOS SENTIDOS DEL COMPROMISO SOCIAL UNIVERSITARIO. UNA APROXIMACIÓN A la construcción del estado del arte. Carina Andrea Gerlero.

\section{El Compromiso Social y la Función Social de Universidad}

En este apartado abordaremos las perspectivas que relacionan al CSU con el rol social de la Universidad. Así, podemos ubicar la concepción de Miguel Rojas Mix (2007) al plantear la necesidad de una educación de calidad para todos, que posibilite formar un "profesional social" comprometido con las situaciones propias de un determinado tiempo y contexto histórico, político y social (2).

En una línea de análisis semejante, y en el marco de un proyecto de investigación sobre Compromiso Social Universitario (3), Cecchi, Lakonich, Perez y Rotstein (2009:11-12) plantean que el CSU es la base para debatir acerca de los objetivos de las Instituciones Universitarias y el modo que utiliza la Universidad Latinoamericana para vincular sus acciones con las problemáticas nacionales y regionales, y dentro de éstas resaltando la de los sectores más desprotegidos. Sitúan el surgimiento de la idea del CSU en Argentina, con la Reforma Universitaria del '18, y analizan que en el país esta idea no se sostiene de manera constante, estando presente en los períodos democráticos y ausente en los períodos regidos por los sucesivos golpes militares. Identifican como otro obstaculizador del CSU, al paradigma científico - tecnológico, lo cual puede pensarse así dado que éste sitúa los roles académicos desvinculados del contexto donde se desarrollan.

Los autores arriba mencionados proponen concebir al CSU desde tres principios: "Educación de Calidad igual para todos" (tomado de la UNESCO), "Finalidad" e "Identidad" (ambos propuestos por Rojas Mix). Sobre la finalidad de la ES universitaria, refieren a qué profesional debe formarse en las aulas universitarias y adhieren, al igual que Rojas Mix, a la idea de un profesional social, que actúe en un marco de solidaridad. En relación a la Identidad de la Universidad, plantean la relación de ésta con la cultura, la paz, la reproducción y perfeccionamiento de un modelo social de integración. Rescatan distintos aportes de Taborda y Freire que facilitan pensar el CSU como:

(...) un modo de escribir el mundo cultural, y significa un modo de 'escribirlo', donde esa escritura -aunque surja de lecturas y escrituras previas, nunca aisladas ni individuales- es un acto creativo, un acto que no se ciñe a un cierre prematuro de los sentidos de la experiencia, la vida y el mundo, sino que inscribe la apertura de otros mundos y experiencias posibles construidos en la relación Universidad - Sociedad (Huergo, 2006 citado por Cecchi et al., 2009, : 41).

Por su parte, Boaventura de Sousa Santos $(2005,: 78)$ plantea que las Universidades deben asumir su RSU aceptando ser permeables a las demandas sociales, principalmente de aquellas provenientes de quienes tienen menos posibilidades y menos poder para imponerlas. Reflexiona acerca de facilitadores de los desafíos de la RSU, tales como la autonomía, la libertad académica y condiciones adecuadas en lo institucional, entendiendo que los desafíos 
Revista de la Escuela de Ciencias de la Educación, año 10, número 9, enero a diciembre de 2014. Páginas 129-146. ISSN 1851-6297. ISSN EN LÍNEA 2362-3349. LOS SENTIDOS DEL COMPROMISO SOCIAL UNIVERSITARIO. UNA APROXIMACIÓN A la construcción del estado del arte. Carina Andrea Gerlero.

contextuales en función de la región, del lugar, no pueden ser enfrentados de manera rígida ni general.

Además, el autor propone que la Universidad debe reconquistar su legitimidad a través de un amplio programa de RSU, que contemple líneas de acción en relación al acceso, a la extensión, a la investigación-acción, a la ecología de los saberes, a la relación Universidad-Escuela Pública y a la relación Universidad e Industria.

A continuación se expone brevemente el análisis de cada una de estas líneas. Se advierte que, desde su posicionamiento, el CS tiene que ver con ir más allá de la masificación, para avanzar en la democratización del acceso. Para este fin se requiere tanto la gratuidad como la asignación de ayudas económicas (becas) para estudiantes de clases trabajadoras; el trabajo conjunto entre las Universidades y las escuelas públicas para promover la integración de ambos sistemas y mejorar las condiciones de enseñanza y de aprendizaje en el campo pedagógico y científico; y medidas de acción afirmativa que confronten la discriminación asociada a condiciones raciales, étnicas o de vulnerabilidad social. Asimismo, otro aspecto central que propone es revisar los conocimientos que se transmiten para evitar concepciones elitistas, meritocráticas y de privilegios en las generaciones presentes y futuras, aquí cobra valor centrar la formación de docentes desde la esfera pública y no desde los principios que promueven muchas instituciones sujetas a modalidades del mercado educativo.

Asimismo, visualiza que el CS emerge cuando se llevan a cabo actividades de extensión que se sustenten ideológica y políticamente en la "construcción de la cohesión social, en la profundización de la democracia, en la lucha contra la exclusión social y la degradación ambiental, y en la defensa de la diversidad cultural." (De Sousa Santos, Ibid.:67). En esta línea de argumentación, cobra sentido la investigación-acción, en tanto prevea e involucre la participación de actores sociales, no universitarios, en su diseño y ejecución. Este autor también entiende que la ecología de los saberes profundiza la investigación-acción al mismo tiempo que actúa como extensión inversa, posibilitando que los saberes provenientes de distintos ámbitos -populares, legos, tradicionales- entren en diálogo con los saberes científicos y humanísticos que la Universidad produce. Estas estrategias pueden dar lugar a una reorientación solidaria de la relación universidad-sociedad.

Por otra parte, señala que los conceptos "sociedad del conocimiento" o "economía basada en el conocimiento" pretenden focalizar la producción de conocimiento desde un paradigma neoliberal, con el fin de favorecer a las grandes empresas, y consecuentemente incrementar sus ganancias. Esta concepción es la que hace del conocimiento un bien privado o privatizable, transable en el mercado. Por el contrario actuar desde el CSU interpela a fijar y regular la agenda de la investigación científica desde los múltiples intereses de la sociedad, posibilitando áreas de investigación más amplias e intercambios entre la 
Revista de la Escuela de Ciencias de la Educación, año 10, número 9, enero a diciembre de 2014. Páginas 129-146. ISSN 1851-6297. ISSN EN LÍNEA 2362-3349. LOS SENTIDOS DEL COMPROMISO SOCIAL UNIVERSITARIO. UNA APROXIMACIÓN A la construcción del estado del arte. Carina Andrea Gerlero.

Universidad y las empresas que no impliquen necesariamente la dependencia académica y la supervivencia económica de las IES.

Otro aporte pertinente a la temática del CSU, que refuerza la idea de su relación con la función social, es el que realizan de la Cruz Ayuso y Sasia Santos (2008), dado que centran la atención del compromiso de la Universidad en la construcción de ciudadanía y en el poder que tiene esta institución para influir en la sociedad y facilitar una acción transformadora de las situaciones de mayor vulnerabilidad social. En este sentido, entienden que la formación de ciudadanos responsables, la contribución al desarrollo social y la generación de nuevos conocimientos contribuyen a la RS pero no se constituyen como objetivos en sí mismo, sino que estos aspectos hacen posible la transformación y construcción de una sociedad, desde la ética y la justicia social. Así, justifican que pensar a la responsabilidad desde el campo de la RSE y desde los conceptos de la gestión empresarial, confunde y acota los alcances del CSU, porque la Universidad no se sostiene desde el principio de la "utilidad" económica ni debe reducir su acción social a acciones asistencialistas o caritativas. Proponen pensar la responsabilidad desde las siguientes dimensiones de análisis, atendiendo a:

Un primer significado consecuencialista, que, en el proceso de toma de decisiones, enfatiza el análisis de las consecuencias y llama a asumir los posibles impactos que se derivan de ese proceso, principalmente los impactos negativos. Un segundo significado contractual, que pone en valor el deber de responder que todos, personas y organizaciones, tenemos como actores o agentes significativos de una sociedad. $Y$ finalmente, un tercer estadio prospectivo que subraya el a priori de ese deber, y la necesidad de pre-ocuparse y hacerse cargo, y que remite a personas u organizaciones que están dispuestas a asumir, a través de sus acciones o de su actividad, la regeneración de nuestras sociedades injustas y desiguales" (de la Cruz Ayuso y Sasia Santos, Ibid.:35).

Así, proponen avanzar hacia el tercer significado de responsabilidad, dado que es el que posibilita entender a la Universidad como una de las instituciones de la sociedad y el consecuente compromiso que éstas deben tener con lo social, así como las modalidades de interacción e intervención en las problemáticas sociales.

Se puede observar que las concepciones hasta aquí expuestas centran su atención en cómo se implica la Universidad, a partir de sus funciones esenciales, en la construcción y transformación de las sociedades de las que son parte. Allí radica la relación entre el CS, la función social y un modelo de universidad progresista, involucrada activamente en las particularidades de su contexto.

Desde allí, los planteos más relevantes tienen que ver con el desafío de formar un profesional social y la necesidad de una reorientación solidaria del vínculo entre la Universidad y la sociedad. Una interacción que, desde un 
Revista de la Escuela de Ciencias de la Educación, año 10, número 9, enero a diciembre de 2014. Páginas 129-146. ISSN 1851-6297. ISSN EN LÍNEA 2362-3349. LOS SENTIDOS DEL COMPROMISO SOCIAL UNIVERSITARIO. UNA APROXIMACIÓN A la construcción del estado del arte. Carina Andrea Gerlero.

marco de diálogo y articulación de políticas, posibilite transformar positivamente las condiciones de vida de los sectores más vulnerables, promueva la convivencia democrática de las ideas y valore los aportes provenientes de la diversidad social y cultural. Es en estos planteos donde se puede observar el modo en que el CSU se vincula con los postulados provenientes de la Reforma Universitaria del ' 18 y la necesidad de profundizar los alcances de los principios reformistas, adaptando las acciones para hacerlas pertinentes a los desafíos del momento actual.

\section{La Responsabilidad Social Universitaria desde la visión empresarial}

En este apartado se repasarán los enfoques que relacionan la RSU con la RSE. En esta línea de análisis, se sitúa la concepción de Torres Pernalete y Trápaga Ortega (2010), quienes establecen un nexo entre el rol social de la universidad (4) y la actual nominación de extensión universitaria, CSU, RSU u otros, pero fundamentalmente ubican los antecedentes más recientes del surgimiento del concepto de RSU a fines de la década del ' 90 , en coincidencia con el recrudecimiento de la globalización. Plantean que el paradigma de la RSU emerge desde la necesidad de un nuevo contrato social de la Universidad con la Sociedad, siendo éste promovido por la Red Universitaria de Ética y Desarrollo Social del BID, la cual implementó el Programa de Apoyo a Iniciativas de RSU, Ética y Desarrollo en el año 2006. Aquí, la RSU se entiende como un concepto expresado en término de gestión de los impactos (5) para las funciones sustantivas de la Universidad: docencia, investigación, extensión y gestión.

Asimismo, las autoras plantean que lo central en relación al concepto de RSU es que configura una conducta ética, de corresponsabilidad con el entorno. En este sentido, entienden que la Universidad, además de formar profesionales y prestar un servicio educativo, es una institución "al servicio de lo que es pertinente para el individuo y para la sociedad en general". Entienden que una Universidad socialmente responsable atiende a las necesidades de la propia universidad y de la sociedad; promueve aprendizajes para el desarrollo humano sostenible; debe preocuparse por sus propias actividades y por la calidad de vida de sus integrantes; debe asumir una posición crítica y de evaluación permanente; debe contar con un órgano de gestión de la RSU.

La concepción de Françoies Vallaeys, de la Cruz y Sasia (s, f) refiere a la RSU como gestión de impactos y rescata los conceptos provenientes de la RSE para su aplicación en la RSU. Definen a la RSU como aquella que "busca resituar a la Universidad en la sociedad, alineando sus cuatro procesos (gestión, docencia, investigación, extensión) con la misión universitaria, sus valores y compromiso social" (:22). Advierten sobre su anclaje con la ética y con las acciones que posibiliten su desarrollo en la práctica.

Sobre los impactos generales que las instituciones universitarias deben gestionar, proponen impulsar un "campus responsable", que promueva una 
Revista de la Escuela de Ciencias de la Educación, año 10, número 9, enero a diciembre de 2014. Páginas 129-146. ISSN 1851-6297. ISSN EN LÍNEA 2362-3349. LOS SENTIDOS DEL COMPROMISO SOCIAL UNIVERSITARIO. UNA APROXIMACIÓN A la construcción del estado del arte. Carina Andrea Gerlero.

cultura organizacional universitaria que rescate y refuerce los derechos laborales, sociales y ambientales, al tiempo que potencien el desarrollo humano y ambiental y aumenten el capital social. En relación a los impactos propios de la Universidad, refieren por una parte a aquellos de carácter educativo, que apuesten a la formación de ciudadanos y profesionales responsables con la sociedad y, por otra parte, a aquellos cognitivos y epistemológicos, que tiendan a la apropiación social del conocimiento e incluyan la participación de actores no académicos en las prácticas de investigación.

Entienden que la RSU precisa y mejora al CSU, porque sitúa a la Universidad en la necesidad de gestión y le aporta herramientas a tal fin, además de promover el diálogo y la participación con distintos actores universitarios y no universitarios.

Por otra parte, Saravia y Escobar (2010) relacionan la RS con la atención de las necesidades de la población, con la ética y los derechos humanos, con el desafío de contribuir al bien común y a la sustentabilidad. Analizan a la RSU como parte de la RS inherente a organizaciones de distinto origen (social, político y económico) y al Estado, identificando que la noción de RS inicialmente surgida para organizaciones empresarias se extendió a otros ámbitos. Consideran también que, actualmente, la globalización de las comunicaciones y los mercados de trabajo requieren del conocimiento, dando lugar a diferencias con anteriores formas de "formación de valor" en el mercado. El valor económico de la información, si bien es intangible, constituye el principal generador de valor económico, así entienden que la justicia social tiene que ver con una distribución equitativa del conocimiento y con la atención de las necesidades del conjunto social.

Margetic y Suarez (2006), analizan lo establecido en los Estatutos de las Universidades Nacionales en lo referente a sus misiones, función social y extensión universitaria, con el propósito de identificar cómo se presentan las modalidades institucionales de compromiso con los problemas nacionales. Observan que en los Estatutos aparece el "deber ser", que tensiona entre aspiraciones de universalidad y de particularidad y que no siempre se condice con las políticas universitarias en curso, asimismo valoran que "este prestar atención a lo propio aparece definido como responsabilidad social, sensibilidad y fundamentalmente como compromiso solidario con la comunidad" (:27).

En las perspectivas expuestas en este apartado, se visualiza que rescatan la necesidad de vincular las acciones de la Universidad con su entorno, para propiciar el desarrollo humano sustentable y la justicia social. Entienden a la RSU como una modalidad que posibilita tal vínculo, pero el acento está puesto en cómo se lleva adelante la gestión de estos impactos en las IES.

Así, los autores plantean la necesidad de implementar procesos y mecanismos de evaluación institucional, de rendición de cuentas, de buenas prácticas organizacionales, de publicidad de las acciones. Entienden que, de esta manera, 
Revista de la Escuela de Ciencias de la Educación, año 10, número 9, enero a diciembre de 2014. Páginas 129-146. ISSN 1851-6297. ISSN EN LINEA 2362-3349. LOS SENTIDOS DEL COMPROMISO SOCIAL UNIVERSITARIO. UNA APROXIMACIÓN A la construcción del estado del arte. Carina Andrea Gerlero.

se establece un contrato social de la Universidad con la sociedad y se construye una gestión ética y una cultura organizacional que tenga en cuenta derechos laborales, sociales y ambientales. Estos conceptos, provenientes del paradigma de la RSE, pueden realizar aportes que fortalezcan la institucionalidad y logren, de esta manera, la concreción de objetivos sociales en las distintas funciones de la Universidad. Pero también se pueden constituir en aquello que ligue sus prácticas a las reglas del mercado, por lo que se requiere una fuerte vigilancia para no desdibujar los objetivos centrales que se pretenden de la RS.

La contribución de la RSU radica en destacar la necesidad de gestionar para transformar. Los proyectos y las acciones de la Universidad requieren de la planificación estratégica, para lograr que efectivamente contribuyan a las finalidades que se proponen. No obstante, el desafío es evitar que la gestión se transforme en un objetivo en sí misma, desnaturalizando su razón de ser, para instituirse como ordenador privilegiado de los objetivos universitarios y, así, llevar a lo educativo hacia un modelo neoliberal.

\section{Reflexiones Finales}

El aporte más importante desde la idea del CSU, entendido como inherente a la función social, es en lo concerniente a ocuparse de los objetivos universitarios en su relación con el afuera de la propia institución, se trata de pensarlos desde la autonomía de la Universidad con la mirada puesta en la sociedad. Desde este posicionamiento, se pone en primer lugar a lo social y a los ciudadanos, con el propósito de transformar las condiciones de vida, de propiciar la inclusión social y construir sociedades más justas y democráticas. Los procesos universitarios no pierden su especificidad, pero se organizan y se desarrollan de manera que las funciones básicas se sostengan en lo propio de un campo disciplinar y en cómo este campo se vincula con su contexto profesional, cultural y social. Así, cobran valor los aportes provenientes del diálogo y la articulación de acciones entre actores políticos, sociales y del estado. Esta idea se sostiene en un modelo humanístico y progresista de educación y de Universidad, que en nuestra identidad latinoamericana, sigue la línea ideológica y política que iniciaron los reformistas de 1918. El gran desafío, es que estos planteos no queden en una declaración de principios, sino que puedan ser posibles en las prácticas cotidianas.

El análisis realizado acerca del campo del CSU, tanto desde los hechos que posibilitaron su surgimiento como desde el aporte de investigaciones y concepciones teóricas, consolida su vínculo indisoluble con la función social de la Universidad. Así, se identifican algunos principios obligados al momento de referirse al CSU, tales como:

- La definición de proporcionar educación universitaria de calidad igual para todos. 
Revista de la Escuela de Ciencias de la Educación, año 10, número 9, enero a diciembre de 2014. Páginas 129-146. ISSN 1851-6297. ISSN EN LÍNEA 2362-3349. LOS SENTIDOS DEL COMPROMISO SOCIAL UNIVERSITARIO. UNA APROXIMACIÓN A la construcción del estado del arte. Carina Andrea Gerlero.

- La democratización y la orientación solidaria de las acciones universitarias, a través de las funciones de docencia, extensión, investigación y gestión.

- La necesidad de establecer un diálogo con la sociedad, con el propósito de contribuir conjuntamente a desarrollar la cultura y los procesos de integración regional, a disminuir la exclusión y brechas de desigualdad, a profundizar la democracia y el bien común.

- Asumir un compromiso ético con la transformación y construcción social.

El planteo de estos principios no son excluyentes, ni se contraponen, a la especificidad de la Universidad -en tanto ámbito de circulación, transmisión y construcción de saberes y conocimientos- se trata de posicionar desde la función social el sentido político de la Universidad. En contraposición a las concepciones que sostienen la neutralidad de los conocimientos y de la ciencia, el CSU implica a las IES, las involucra, les posibilita ser parte de los procesos sociales, políticos, económicos y culturales. Ser parte no debe interpretarse como sinónimo de ser la única que debe hacerse cargo de estos procesos, significa que no puede pensarse alejada de ellos.

Asimismo, el CSU involucra desafíos que se sitúan al interior de los procesos universitarios, y aquí lo que se pone en juego es el sentido político de la educación. De identificar si se trata de proporcionar un conjunto de conocimientos relacionados con determinadas herramientas técnicas que permitan acceder a los procesos productivos o de prestación de servicios o se trata desarrollar la cultura y propiciar la apropiación de bienes sociales y culturales o de una combinación de ambos.

Intentar reconstruir los sentidos del CSU deja entrever que existen diversos conceptos polisémicos que, cuando se los utiliza en relación al CSU, generan dilemas acerca del significado que se le concede, porque también se relacionan con el sentido político de Universidad y de la educación. Igualmente, esta diferencia en la asignación de significados, puede dificultar la identificación del posicionamiento ideológico que sostiene y da identidad a determinados conceptos, tales como pertinencia, misión de la Universidad, gestión, autonomía universitaria, libertad académica, entre otros.

En esta línea, los dilemas se trasladan a la misión de la Universidad y al objetivo de la gestión universitaria: ¿se trata de contar con una metodología de trabajo que sitúe los propósitos políticos y democráticos y delimite los pasos a seguir para alcanzar esa intencionalidad?, ¿o se piensa a la gestión con un objetivo en sí misma, para el control, la verificación, la rendición de cuentas?. Igualmente, la autonomía y la libertad académica tienen en sus extremos defensores y opositores intransigentes, desde quienes ven en ellas la posibilidad de mantener a la Universidad alejada de toda influencia que provenga del afuera, hasta aquellos posicionamientos que las piensan como lo que hace posible el vínculo con la sociedad. 
Revista de la Escuela de Ciencias de la Educación, año 10, número 9, enero a diciembre de 2014. Páginas 129-146. ISSN 1851-6297. ISSN EN LÍNEA 2362-3349. LOS SENTIDOS DEL COMPROMISO SOCIAL UNIVERSITARIO. UNA APROXIMACIÓN A la construcción del estado del arte. Carina Andrea Gerlero.

En definitiva, cada uno de estos planteos exterioriza las concepciones políticas, las ideologías que subyacen, ya sea en el contexto macro como el micro en que se desarrollan. El desafío más potente probablemente lo constituya el poder despejar lo que aparece en la superficie de aquello que está en la profundidad de cada concepto. Poder trascender la confrontación de ideas partiendo de aquello que "creemos" que el otro sostiene, por la identificación de la ideología que efectivamente se presenta en la configuración de un determinado discurso, más allá de los términos que se usen.

Nos dice Hanna Arendt (1997) que "si entendemos por político un ámbito del mundo en que los hombres son primariamente activos y dan a los asuntos humanos una durabilidad que de otro modo no tendrían, entonces la esperanza no es en absoluto utópica" (:50). Las Universidades y los universitarios tienen mucho que ofrecer en relación a aportes que posibiliten este anclaje con lo ideológico y con lo político. Desde el CSU, entendido como la expresión de la función social universitaria, se desprenden líneas de acción tendientes a democratizar el acceso, el currículum, la investigación, la transferencia de conocimientos. Asimismo, a través del CSU se pueden revalorizar y expandir el concepto de extensión, fundamentalmente en términos de cómo se posiciona frente al otro no universitario, en trabajar sobre lo que nos hace ciudadanos, sobre aquello que determina hoy la inclusión y la exclusión, en el cómo se ponen en juego valores que tengan en cuenta lo colectivo en una sociedad sustentada en la competencia individual y en los valores económicos.

Otro aspecto interesante a destacar son las prácticas que desarrollan las IES desde la perspectiva del CS. En el presente trabajo no se profundizó en esta dimensión, dado que el recorte realizado se centró en los principios o concepciones teóricas que sostienen al CSU. No obstante, se encuentran disponibles numerosos materiales bibliográficos que se centran en el registro y análisis de prácticas que, desde fundamentos afines al CS, son llevadas adelante en distintos ámbitos académicos. Este hecho, refuerza la idea de que se trabaja sobre la caracterización de un campo aún en construcción, donde queda mucho por hacer y por conceptualizar. Por otra parte, se trata de un campo que se construye desde lo académico, pero también desde la gestión y desde la práctica de los actores, en este sentido es un campo distinto a otros porque está mucho más permeado por las prácticas, por las definiciones institucionales y por aspectos geopolíticos. Avanzar en un análisis de este orden, seguramente contribuirá a dar mayor precisión al campo y a los sentidos del CSU. De igual manera, podría hacer visibles los modos en que se canalizaron los llamados a la acción de la CRES y de la CMES, las líneas de acción concretas que implementaron las IES y en qué medida pudieron transformarse las políticas públicas institucionales en materia de docencia, investigación y extensión.

Los desafíos que enfrenta la ES en el contexto actual, ante las consecuencias culturales de la globalización y de los cambios en las estructuras 
Revista de la Escuela de Ciencias de la Educación, año 10, número 9, enero a diciembre de 2014. Páginas 129-146. ISSN 1851-6297. ISSN EN LINEA 2362-3349. LOS SENTIDOS DEL COMPROMISO SOCIAL UNIVERSITARIO. UNA APROXIMACIÓN A la construcción del estado del arte. Carina Andrea Gerlero.

sociales, pareciera muchas veces llevarnos a un terreno donde ya no queda nada por decir o por hacer. Sin embargo, desde el modelo que imprimió en las Universidades Latinoamericanas la Reforma del '18, hoy nos sigue convocando el ser parte de un cambio progresista en el estado y en la sociedad, que desde la defensa de la vida democrática y la paz, posibilite la reconstrucción de lazos solidarios y revalorice la identidad y la cooperación entre los países de nuestra región.

Asumir el CSU desde la función social de las universidades contribuye a instituir legitimidad social en la ES, al mismo tiempo que posibilita organizar los procesos universitarios de manera que las acciones se sostengan desde lo propio de un campo disciplinar y desde el vínculo del campo con su contexto profesional, cultural y social. De esta manera la Universidad mantiene su esencia y, desde la autonomía, establece procesos de intercambio con la sociedad.

\section{Notas Bibliográficas}

(1) El artículo comunica parcialmente el Trabajo Final elaborado para acceder al título de Especialista en Política y Gestión de la Educación Superior en la Universidad Nacional de Rosario, aprobado el 28 de mayo de 2014. Asimismo contribuye a la formulación definitiva y al desarrollo del Plan de Tesis Doctoral: "La formación de profesionales desde la perspectiva del compromiso social universitario", a realizar en el Doctorado en Educación en Ciencias Experimentales, de la Facultad de Bioquímica y Ciencias Biológicas de la Universidad Nacional del Litoral (UNL). El trabajo de campo de la Tesis Doctoral se realizará en el marco del Proyecto "La formación de profesionales desde la perspectiva del compromiso social. Huellas y olvidos", aprobado por la UNL en el CURSO DE ACCIÓN PARA LA INVESTIGACIÓN Y EL DESARROLLO "Programa de I+D Orientado a Problemas Sociales y Productivos". Convocatoria 2012.

(2) El posicionamiento de Miguel Rojas Mix se tuvo en cuenta durante el análisis de los aportes del Congreso de Rectores, por lo cual no se profundiza en este apartado.

(3) El mencionado Proyecto de Investigación es sobre "Compromiso Social Universitario" y se desarrolla en el marco del Instituto de Estudios y Capacitación de la Federación Nacional de Docentes Universitarios (CONADU), donde se sitúa un Área de Investigación cuyo objeto de estudio es la Universidad.

(4) A su vez, las autoras sitúan el origen del rol social de la Universidad en la Reforma Universitaria del '18.

(5) Las autoras citan los impactos que debe producir la aplicación de la RSU, de acuerdo a lo expresado por Vallaeys: Gestión socialmente responsable de la organización y sus recursos; de la formación académica y pedagógica; de la producción del saber y los modelos epistemológicos promovidos; y de la participación en el DHS. Torres Pernalete y Trápaga Ortega (2010:79).

\section{Referencias Bibliográficas}

- $\quad$ Arendt, H. (1997). ¿Qué es política?, Barcelona: Paidós.

- $\quad$ Cecchi, N., Lakonich, J., Pérez, D. y Rotstein, A. (2009). El Compromiso Social de la Universidad Latinoamericana del Siglo XXI: Entre el debate y la acción, IEC-CONADU 
Revista de la Escuela de Ciencias de la Edducación, año 10, número 9, enero a diciembre de 2014. Páginas 129-146. ISSN 1851-6297. ISSN EN LINEA 2362-3349. LOS SENTIDOS DEL COMPROMISO SOCIAL UNIVERSITARIO. UNA APROXIMACIÓN A la construcción del estado del arte. Carina Andrea Gerlero.

y ADUM. Recuperado de:http://www.iecconadu.org.ar/images/LIBRO\%203\%20EI\%20 compromiso\%20Social\%20FINAL.pdf

- Conferencia Mundial de Educación Superior (2009): Las nuevas dinámicas de la educación superior y de la investigación para el cambio social y el desarrollo. Comunicado Final. Recuperado de: http://www.unesco.org/education/WCHE2009/comunicado_es.pdf

- Conferencia Mundial de Educación Superior (1998). Comunicado final. Recuperado de: http://unesdoc.unesco.org/images/0011/001163/116345s.pdf

- $\quad$ Conferencia Regional de Educación Superior en América Latina y el Caribe (2008). Declaración final. Recuperado de: http://www.cres2008.org/es/index.php

- Conferencia Regional sobre Políticas y Estrategias para la Educación Superior en América Latina y el Caribe (1996). Proclamación final. Recuperado de: http://www.rau. edu.uy/docs/havdecs.htm

- Congreso Internacional de Rectores Latinoamericanos y Caribeños (2007). Declaración final. Recuperado de: http://www.unesco.org.ve/dmdocuments/3encuentro/ DeclaraCongRectores_Esp.pdf.

- $\quad$ De la Cruz Ayuso, C. y Sasia Santos, P. (2008). La responsabilidad social de la universidad en el proyecto de construcción de una sociedad. Revista Educación Superior y Sociedad. El movimiento de responsabilidad social de la universidad: una comprensión novedosa de la misión universitaria, 13(2). Recuperado de: http://ess.iesalc.unesco.org. ve/index.php/ess/issue/view/5

- De Sousa Santos, B. (2005). La Universidad en el Siglo XXI. Para una reforma democrática y emancipadora de la Universidad, México: Centro de Investigaciones Interdisciplinarias en Ciencias y Humanidades, Universidad Nacional Autónoma de México.

- Garcia Guadilla, C. (2008). El compromiso social de las universidades [versión electrónica]. Cuadernos del Cendes, 25(67), 129-134.

- Margetic, A. y Suarez, V. (2006). La función social de la Universidad. Consideraciones acerca de los Estatutos de las Universidades Nacionales. Remedios de Escalada: UNLa.

- Saravia, F. y Escobar, J. (2010). Hacia una democracia socialmente responsable. Una reflexión desde la Universidad Pública. $1^{\circ}$ edición, Buenos Aires: Prometeo.

- Rojas Mix, M. (2007). El Compromiso Social de las Universidades de América Latina y el Caribe. Recuperado de: http://ess.iesalc.unesco.org.ve/index.php/ess/article/view/46/33

- Torres Pernalete, M. y Trápaga Ortega, M. (2010), Responsabilidad Social de la Universidad. Retos y Desafíos. $1^{\circ}$ edición, Buenos Aires: Paidós.

- Vallaeys, F.; de la Cruz, C. y Sasia, P. (s, f), Manual de primeros pasos en responsabilización social universitaria. Construyendo ciudadanía en universidades responsables. Recuperado de: http://www.cyta.com.ar/biblioteca/bddoc/bdlibros/rse/334_as_manual_rsu_bid.pdf. 